\title{
Numerical investigation of hybrid blend design target bullets
}

\author{
$V R$ Muruganantham ${ }^{1}, T$ Babin $^{2 *}$ \\ ${ }^{1,2}$ Dept. of Mech. Engineering, Kumaraguru College of Technology, Coimbatore, Tamil Nadu, India
}

\begin{abstract}
In the past few decades, researchers are involved in the studies of high-energy penetrator geometry and also the materials used for causing brutal damages on enemy targets. The highly focused area in this field is the shape of the nose and penetration angle of the penetrator. Hybrid blend design target bullets is one among the best aerodynamic design as it is having two different shapes within the nose. The design of these bullets plays an influential role in terms of aerodynamic characteristics and its dynamic performance capability on hitting the targets. Objective of this research is to perform a comparative analysis of 3 different geometrically varied shapes of these bullets by numerical simulation using CFD, based on its performance characteristics such as aerodynamic co-efficients, shock wave existence, Turbulent dissipation rate and normal drag force on the bullet surface.
\end{abstract}

\section{Introduction}

One of the exciting challenges for shooters is getting precision and accuracy over the targets. Utilization of technology leads to researches in guns and ballistics to develop such bullets. According to recent researches, Very Low Drag (VLD) bullets have shown significant improvement because of its low drag and efficient performance under high winds. In addition, VLD bullets have very high Ballistic Co-efficients (BCs). Bryan Litz [1], a Ballistician found that these VLD bullets are highly sensitive to seating depth. This is due to the nose design it has, and further researches on the nose ogive design leads to development of hybrid types of bullets. The advantage over the combined tangent and secant ogive design is lies in the improvement of accuracy for long-range shooters on targets. The reason behind the improvement of hybrid bullets is the combined strength of tangent and secant ogive design, in which the tangent ogive from bearing surface of bullet has minimal sensitive to seating depth, whereas the continuous secant ogive gives the high $\mathrm{BC}$ and also very less drag similar to VLD type bullets. In the current research series, this paper contributes the numerical investigation of hybrid blend design target bullets to obtain its aerodynamic characteristics and comparative performance analysis.

\subsection{Geometry}

\subsubsection{Secant-tangent Ogives}

Ogive is the term, which describes the point from where the curve approaches the bullet diameter. Ogive is usually characterised by its length of arc radius. For, a constant nose length, ogive can be of tangent curve or secant curve. If the ogive is said to be perfectly tangent, then the curve is defined by its own very specific radius. And, if the radius is longer than that, then the curve will obtain secant ogive. Secant ogive is usually varied from tangent radius to very long radius. With reference to Bryan Litz [1], it is to be noted that the secant ogive results minimal drag particularly when the radius is twice the tangent ogive radius. Pictorial representation of both curves are given in the Figure 1.
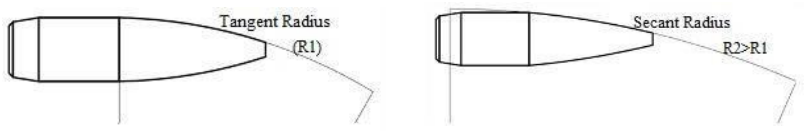

Fig. 1. Tangent ogive vs. Secant ogive.

\subsubsection{Design of Hybrid Target Bullet}

Hybrid blend design bullets for target hunting applications are developed first by Bryan Litz [1] after his research on substantial effect of sensitivity over seating depth of secant ogive type bullets and its target accuracy. On continuous improvement, the combination of tangent ogive into the secant ogive came into existence, which results in very less sensitivity on seating depth and low drag. From bearing surfaces, tangent ogive starts the curve and subsequently secant ogive follows as shown in the figure 2 .

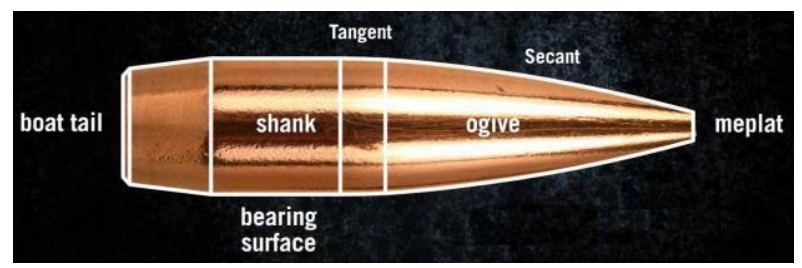

Fig. 2. Hybrid blend design bullet nomenclature.

\footnotetext{
*Corresponding author: babinn4340@gmail.com
} 


\subsection{Model Specification}

Three cases of hybrid bullets are taken for CFD analysis from Berger reference sheet [2]. Dimensional specifications measured in inches for the three cases are discussed in the Table 1.

Table 1. Bullet dimensions for three cases

\begin{tabular}{|c|c|c|c|}
\hline Type/description & $\begin{array}{c}6.5 \mathrm{~mm} \\
140 \mathrm{gr}\end{array}$ & $\begin{array}{c}7 \mathrm{~mm} \\
180 \mathrm{gr}\end{array}$ & $\begin{array}{c}30 \mathrm{Cal} \\
230 \mathrm{gr}\end{array}$ \\
\hline Bullet Dia. & 0.264 & 0.284 & 0.308 \\
\hline Boat Tail & 0.224 & 0.245 & 0.280 \\
\hline Nose Length & 0.768 & 0.852 & 0.859 \\
\hline Base to Ogive & 0.704 & 0.468 & 0.875 \\
\hline Bearing Surface & 0.415 & 0.430 & 0.529 \\
\hline Sectional Density & 0.287 & 0.319 & 0.346 \\
\hline
\end{tabular}

\section{Methodology}

Computational Fluid Dynamics is the tool for solving the set of PDEs governing the fluid motion by numerical methods. Among various commercial packages, ANSYS FLUENT 18.2 (2D) is chosen for numerical simulation of hybrid blend design target bullets. Graphical representation of aerodynamic properties are obtained from CFD POST 18.2. Numerical simulation has been carried out for different speeds ranging from subsonic to supersonic, in which the fluid is assumed to be taken as compressible medium.

\subsection{CFD Modelling and Meshing}

Far-field boundary around the bullets is chosen as flow field for external compressible flow analysis. Parabolic domain of $11 \mathrm{~m}$ extent is developed around the bullet, which is $5 \mathrm{~m}$ far from the flow entry. Discretization of domain was made with the help of ICEM CFD 18.2 for $\mathrm{O}$-grid meshing. To get better accuracy, O-grid mesh is chosen and elements are counted around 1, 65,000 after grid-independent study. $\mathrm{Y}+$ value to capture boundary layer influence on aerodynamic performance is estimated and found to be $0.000481 \mathrm{~mm}$. Figure 3. Shows the meshed domain with full-view of bullet.

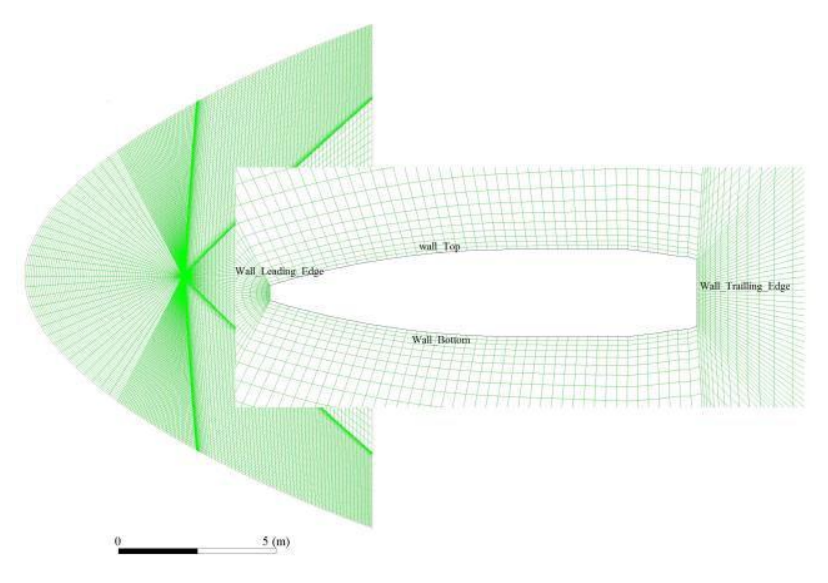

\subsection{Governing Equations}

Governing equations are the PDEs containing fluid variables for solving the fluid flows. One of the most recently developed model, especially for compressible external fluid flow applications by Spalart-Allmaras [3] called one-equation turbulence model is used for CFD analysis. This model was designed specifically for wall bounded aerodynamic flows and shows better accuracy for inflation layers. The one-equation turbulence model [3] is given by the following equation (1):

$\frac{\partial}{\partial t}(\rho \tilde{\nu})+\frac{\partial}{\partial x_{i}}\left(\rho \tilde{\nu} u_{i}\right)=G_{\nu}+\frac{1}{\sigma_{\tilde{\nu}}}\left[\frac{\partial}{\partial x_{j}}\left\{(\mu+\rho \tilde{\nu}) \frac{\partial \tilde{\nu}}{\partial x_{j}}\right\}+C_{b 2 \rho}\left(\frac{\partial \tilde{\nu}}{\partial x_{j}}\right)^{2}\right]-Y_{\nu}+S_{\tilde{\nu}}$

Where, $G_{v}$ is the Turbulent Viscosity Production term $\mathrm{Y}_{\mathrm{V}}$ is the Destruction term (Shear flow region) And, $\mathrm{S}$ is the scalar measure of deformation Tensor.

According to Eleni. D. C [4], the Spalart-Allmaras shown greater accuracy over the experimental results comparing to other two-equation turbulence models. Hence, this model can be used 2D compressible external fluid flow applications. With one-equation model, energy equation is enabled to study the temperature gradient effects because of high Reynold's number.

\subsection{Boundary Conditions and simulation Methodology}

For Compressible fluid flow analysis, reference pressure is taken as zero, so that the corresponding gauge pressure (equal to total pressure) is applied on the far-field condition. Mach number is the major boundary condition, which is obtained from article by Petzal [4] and flow type is categorised. Ideal gas is chosen as material for flow domain. Three coefficient Sutherland law of viscosity model is well suited for high Reynolds number compressible flows. To get faster convergence, implicit scheme is used rather than going for explicit scheme. In addition, Coupled second order upwind scheme is applied especially for applications dealing with high-speed aerodynamics. Table 2. Shows the boundary values and solver schemes used for modelling simulation of hybrid blend design bullets.

Table 2. Boundary conditions and Solution Methodology.

\begin{tabular}{|c|c|}
\hline & Value/ Method \\
\hline Gauge Pressure & $101325 \mathrm{~Pa}$ (Ref. Pressure $=0 \mathrm{~Pa})$ \\
\hline \multirow{3}{*}{ Mach Number } & 0.54 (Subsonic Flow) \\
\cline { 2 - 2 } & 0.90 (Transonic Flow) \\
\cline { 2 - 2 } & 2.27 (Supersonic Flow) \\
\hline Solver & Density-Based, Steady \\
\hline Formulation scheme & Implicit, coupled \\
\hline Material & Ideal-gas \\
\hline Viscosity model & Sutherland \\
\hline Temperature & $300 \mathrm{~K}$ \\
\hline Convergence criteria & $1 \mathrm{e}^{-05}$ \\
\hline
\end{tabular}

Fig. 3. Discretization of domain. 


\section{Results and Discussion}

Results are taken with the convergence of 1E-05, an approximate of 15,000 iterations to achieve such convergence. Numerical simulations helps to visualise the flow distribution over the domain and to plot the technical parameters along the co-ordinate to compare the results. Numerical simulations has been conducted for three different cases at three Mach zones. For aerodynamic performance comparison, drag force is estimated and the velocity contour plots were taken for observing the flow distribution around the bullet. Similarly, the turbulent dissipation contour plots based on vorticity are taken and discussed.

\subsection{Velocity Distribution}

\subsubsection{Subsonic Speed (0.54 Mach)}

The velocity contour graph of three cases at subsonic speed of 0.54 Mach for all three cases are presented in the Figures 4, 5 and 6 . The plots clearly describes the pattern of wave zones near top and bottom edges of bullet. Since, three cases exhibit similar hybrid ogive profile, the flow pattern remains same. However, as far as the size of bullet increases, area covering wave zone and wake zone length increases.

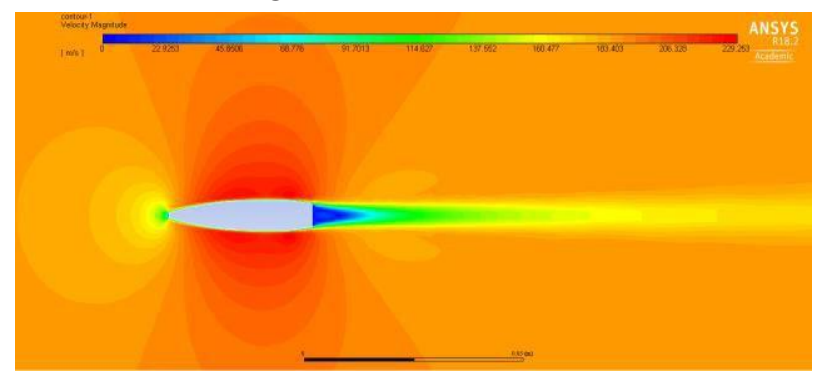

Fig. 4. Velocity contour of first case

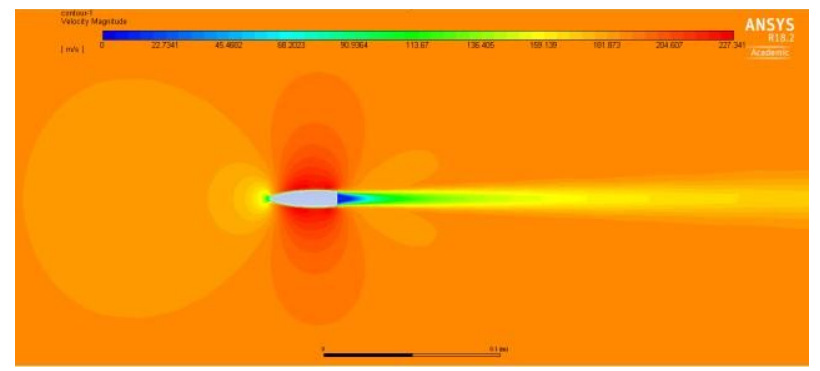

Fig. 5. Velocity contour of second case

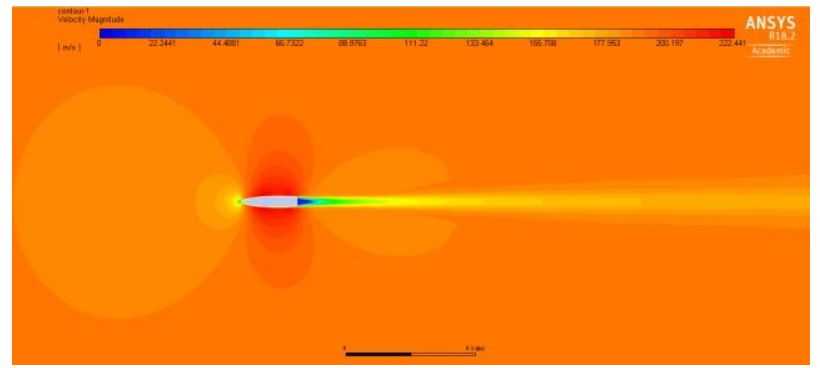

Fig. 6. Velocity contour of third case

\subsubsection{Transonic Speed (0.90 Mach)}

Since, they attain similar flow distribution for the three cases, the second case alone presented for transonic speed in the Figure 7. At Transonic speed, the shock wave region was formed at the top and bottom walls, it shows the transition from subsonic to supersonic speed.

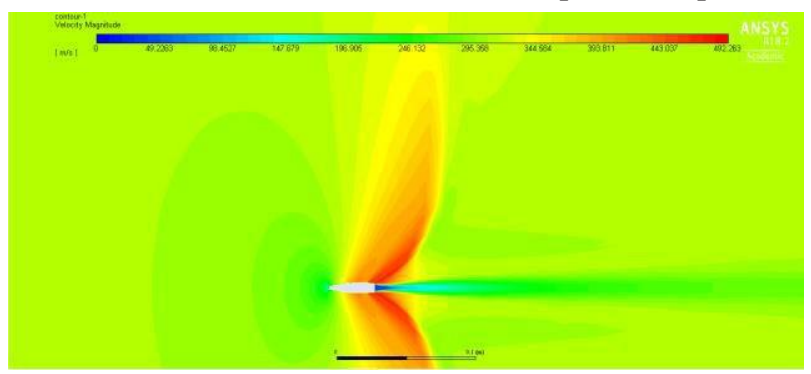

Fig. 7. Velocity Contour of second case (0.90 Mach)

\subsubsection{Supersonic Speed (2.27 Mach)}

On moving to high-speed case, the contour graph in the Figure 8. Shows the supersonic wake existence around the bullet in the direction of flow.

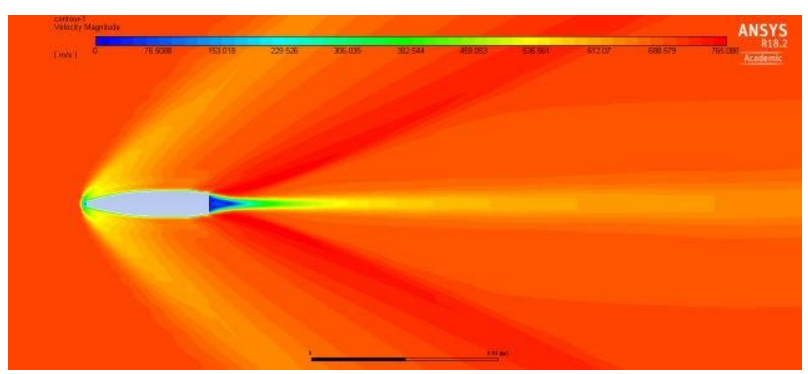

Fig. 8. Velocity contour of second case (2.27 Mach)

\subsection{Aerodynamic forces and co-efficients}

Drag force $\left(F_{d}\right)$ is the force acting normal to the surface of bullet, which is a function of speed and the surface area of contact. Figure 9. Compares the drag force against Mach number for all three cases. Since, the drag force is directly proportional to square of velocity as expressed in following relation (2), the $\mathrm{F}_{\mathrm{d}}$ increases.

$$
F_{d}=\frac{1}{2} \rho v^{2} C_{d} A
$$

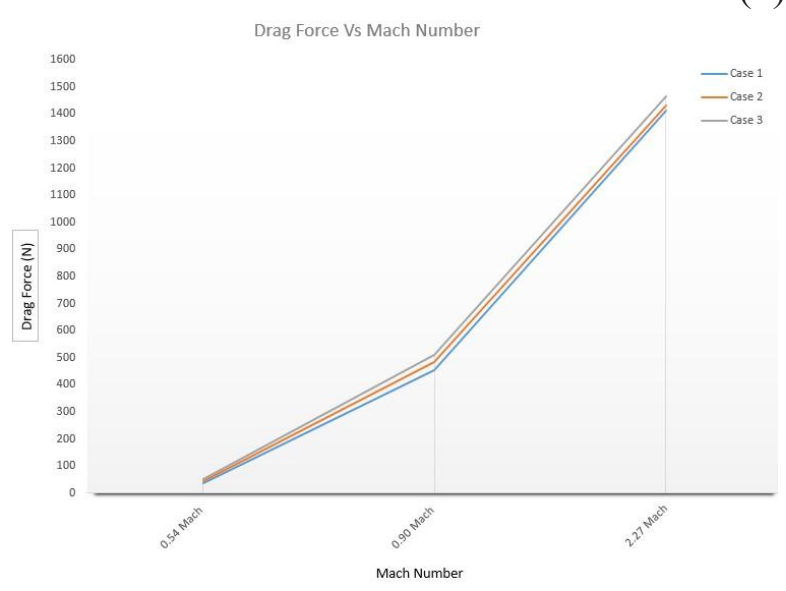

Fig. 9. Drag force Vs Mach Number 
The following relation (3) gives co-efficient of skin friction for the fully turbulent and compressible fluid flow.

$$
C_{D_{f r}}=\frac{0.455\left(\log _{10} \mathrm{Re}\right)-2.58 S_{\text {wet }}}{\left(1+0.21 M^{2}\right)^{0.467} S_{\text {ref }}}
$$

$\mathrm{C}_{\mathrm{D}}$ due to friction is completely dependent of Reynolds number and Mach number for a given profile. Figures 10,11 and 12 presents the co-efficient of skin friction for hybrid blend design bullets at three different Mach number. It clearly shows that the value of skin friction drag increases with increase in Mach number.

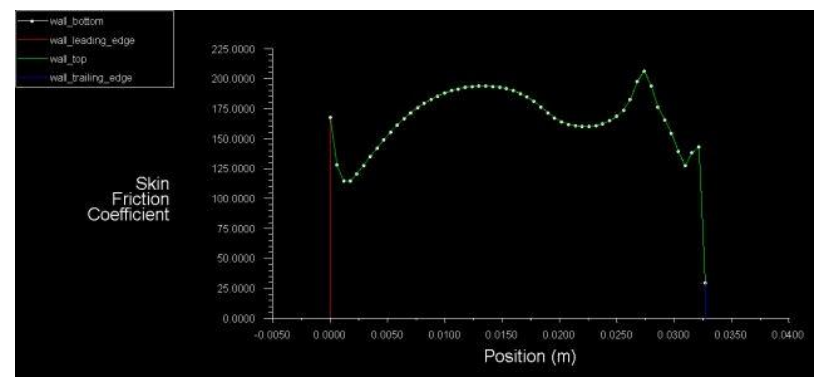

Fig. 10. Skin-friction coefficient at $0.54 \mathrm{Mach}$

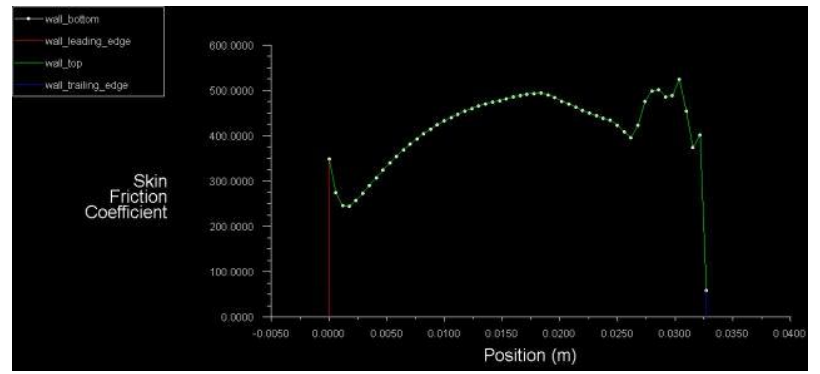

Fig. 11. Skin-friction coefficient at $0.90 \mathrm{Mach}$

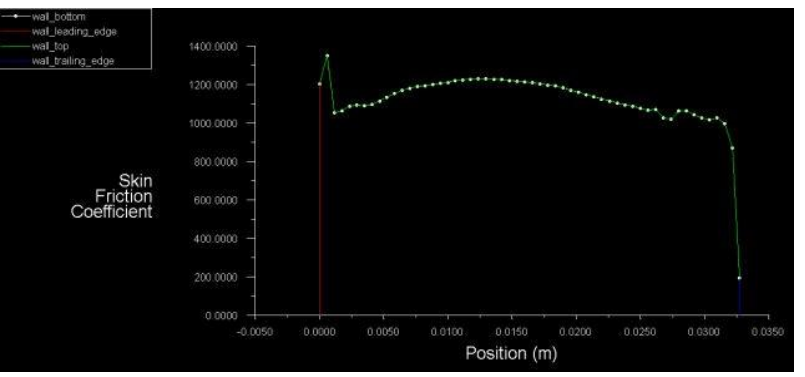

Fig. 12. Skin-friction coefficient at 2.27 Mach

\subsection{Turbulence Properties}

Turbulence produced behind the bullet is a major influence on the performance of bullet. Turbulence viscosity ratio determines the length of its influence behind the bullet.

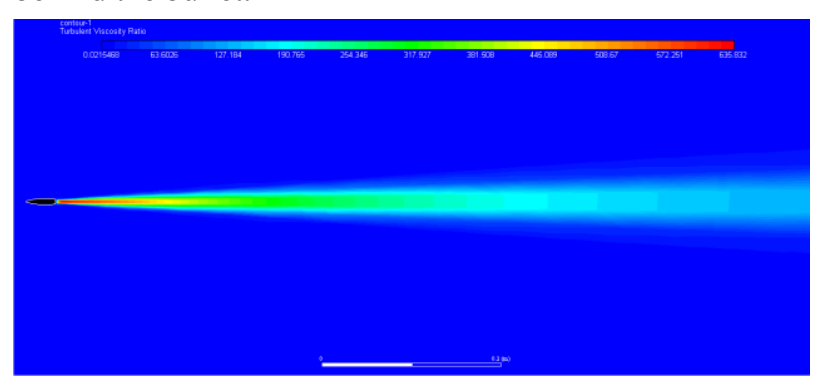

Fig. 13. Viscosity ratio at $0.54 \mathrm{Mach}$

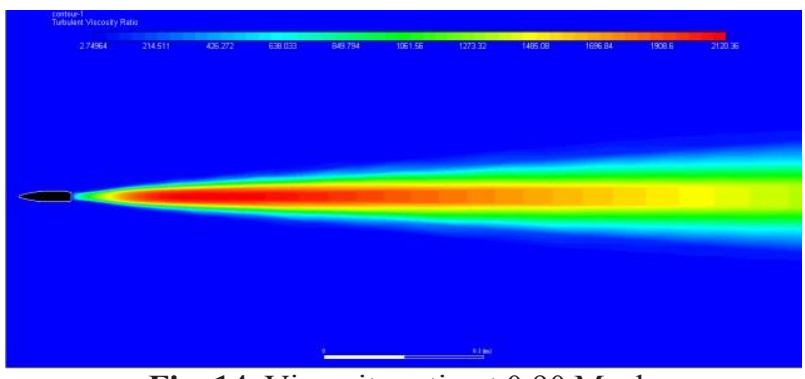

Fig. 14. Viscosity ratio at $0.90 \mathrm{Mach}$

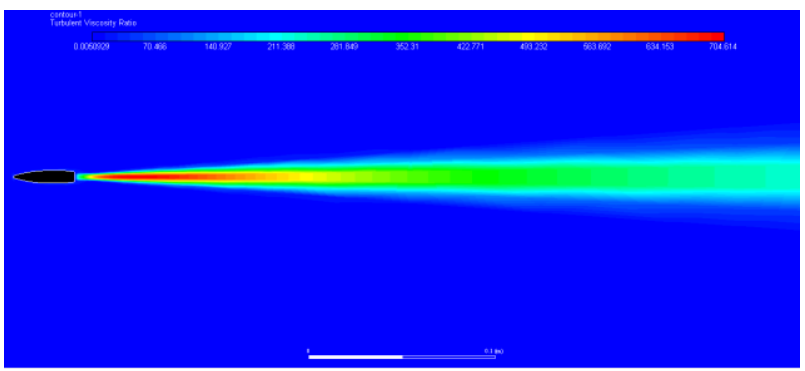

Fig. 15. Viscosity ratio at $2.27 \mathrm{Mach}$

Figures 13, 14 and 15 shows the viscosity ratio at different Mach numbers. It is to be noted that, turbulence produced is more in case of transonic speed. Due to shock wave existence, the turbulence is produced at greater influence.

\subsection{Conclusion}

Three cases of hybrid blend design bullets had been taken and numerical investigation has been carried out. Flow distribution, drag force, skin friction coefficient was monitored and turbulence properties are also investigated. Key findings of this research are discussed below:

a) Velocity profile remains constant for the three different cases. It shows that, for hybrid ogive profile, velocity distribution is independent of size of bullet.

b) Drag force increases with increase in Mach number and it proves the drag force relation.

c) Skin-friction drag increases correspondingly as Mach number increases. Profile of skin-friction coefficient has been validated against mathematical relation.

d) Turbulence effect is almost three times larger in case of transonic speed comparing to subsonic and supersonic conditions.

\section{References}

1. Litz, Bryan. Characteristics of Different Bullet Ogive Designs. App. Ballistics. ABDOC-2.1 (2015)

2. Berger. Bullet Product Catalog. Mesa, AZ (2018)

3. P. R. Spalart, S. R. Allmaras. A One Equation Turbulence Model for Aerodynamic Flows. AIAA92-0439 (1992)

4. Petzal, David E. How Fast is the Speeding Bullet. Field and Stream 97 (1992) 